\title{
Non-Markovian Fokker-Planck equations and turbulent diffusion in plasmas
}

\author{
A.Zagorodny ${ }^{1}$, J.Weiland ${ }^{2}$ \\ 1 Bogolyubov Institute for Theoretical Physics, 252143 Kyiv 143, Ukraine \\ 2 Institute for Electromagnetics, Chalmers University of Technology, \\ S-41296 Göteborg, Sweden
}

Received May 6, 1998

Non-Markovian generalization of the Fokker-Planck equation is proposed in attempt to describe the influence of memory effects on stochastic particle motion in both velocity and configuration spaces. General relations between the time-nonlocal diffusion coefficient, its conventional part and meansquare displacements are established. Application of the proposed non-Marcovian equation to the description of diffusive processes in the configurational space directly leads to a time-nonlocal diffusion equation. The results obtained are in agreement with the continuous time random walk theory.

Microscopic derivation of the non-Markovian equation is given for the specific case of turbulent plasma using particle propagators renormalized with regard for the evolution of kinetic coefficients. The influence of memory effects on transport processes in plasmas with saturated turbulence is discussed.

Key words: Fokker-Planck equation, turbulence saturation, time-nonlocal diffusion

PACS: 05.40. $+j ;$ 52.35.Ra; 25.65.Ff

\section{Introduction}

Recently, much interest has been given to the studies of physical systems with statistical properties which cannot be described within the concept of the Markovian random processes. This concerns anomalous transport in turbulent plasmas [1-3], "strange" spatial diffusion of magnetic field lines [2], the Brownian motion of macroparticles in complex fluids $[4,5]$, etc. One of the most important problems here is to describe the kinetic properties of such systems and to understand the role of the non-Markovian effects in transport phenomena. The purpose of this paper is to work out a theory of potential electric fluctuations in turbulent plasmas 
with regard to the memory effects associated with large scale turbulent perturbations, to calculate the kinetic coefficients, and to estimate the diffusion level for saturated turbulence.

We start from the qualitative approach to the description of memory effects in statistical systems. Within this approach non-Markovian Fokker-Planck equations can be introduced (section 2). A microscopic derivation of the kinetic equation with a non-Markovian collision term is given in section 3 for the specific case of a plasma system. The kinetic coefficients are calculated in terms of the particle transition probability renormalized with regard to the turbulent field influence on the particle trajectories. Equations for the diffusion coefficients in the velocity and real spaces are given in section 4 . In the particular case of a stationary Markovian limit these equations are reduced to the results of the Dupree-Weinstock theory. The results of the extended resonance broadening theory [1] could be also reproduced in the case of a fast growing diffusion coefficient at the initial stage of turbulence saturation.

Some applications of the proposed theory are also discussed. In particular, in the real space we again reproduced the non-Markovian diffusion coefficient which was originally estimated in [6] and previously derived by a somewhat less systematic method in [3].

\section{Generalized Fokker-Planck equations. General approach}

The conventional Fokker-Planck equation is usually derived (see, for example, [7]) from the relation for the Markovian probability distribution $f(X, t)$ given by

$$
f(X, t+\tau)=\int \mathrm{d} \Delta X P(X-\Delta X, \Delta X ; \tau) f(X-\Delta X, t),
$$

where $P(X, \Delta X, \tau)$ is the probability of the particle displacement $\Delta X$ from the starting point $X$ during time $\tau$.

Expansion of equation (1) in $\tau$ with the accuracy up to the first order leads to the equation

$$
\begin{aligned}
\frac{\partial f(X, t)}{\partial t} & =\int \mathrm{d} \Delta X \frac{P(X-\Delta X, \Delta X, \tau)-P(X-\Delta X, \Delta X, 0)}{\tau} f(X-\Delta X, t) \\
& =\left.\int \mathrm{d} \Delta X \frac{\partial P(X-\Delta X, \Delta X, \tau)}{\partial \tau}\right|_{\tau \rightarrow 0} f(X-\Delta X, t) .
\end{aligned}
$$

Expanding then the right-hand part of equation (2) in $\Delta X$ with the accuracy up to the second order one comes to the Fokker-Planck equation

$$
\frac{\partial f(X, t)}{\partial t}=\frac{\partial}{\partial X_{i}}\left\{-A_{i}(X) f(X, t)+\frac{1}{2} \frac{\partial}{\partial X_{j}}\left(B_{i j}(X) f(X, t)\right)\right\},
$$

where

$$
\begin{aligned}
A_{i}(X) & =\left.\frac{\left\langle\Delta X_{i}\right\rangle_{\tau}}{\tau}\right|_{\tau \rightarrow 0}=\left.\frac{\partial\langle\Delta X\rangle_{\tau}}{\partial \tau}\right|_{\tau \rightarrow 0}, \\
B_{i j}(X) & =\left.\frac{\left\langle\Delta X_{i} \Delta X_{j}\right\rangle_{\tau}}{\tau}\right|_{\tau \rightarrow 0}=\left.\frac{\partial\left\langle\Delta X_{i} \Delta X_{j}\right\rangle_{\tau}}{\partial \tau}\right|_{\tau=0}
\end{aligned}
$$


We can see that equation (2) is exact in the limiting case $\tau \rightarrow 0$. It does not mean, however, that the displacement probability $P(X, \Delta X, \tau)$ describes microscopic particle transitions. Actually, $\tau \gg \tau_{\text {cor }}$, where $\tau_{\text {cor }}$ is the correlation time of forces producing a random particle motion. In other words, the limit $\tau \rightarrow 0$ should be treated in the "kinetic" but not "microscopic" sense.

In the case of a nonstationary spectrum of random forces the transition probability $P$ depends on the initial and final times $t$ and $t^{\prime}$, rather than on the time interval $\tau$. This means that equation (1) should be rewritten as

$$
f(X, t)=\int \mathrm{d} \Delta X P\left(X-\Delta X, \Delta X, t, t^{\prime}\right) f\left(X-\Delta X, t^{\prime}\right)
$$

In this case an equation of type (2) can be derived from equation (4) by taking the time derivative

$$
\frac{\partial f(X, t)}{\partial t}=\int \mathrm{d} \Delta X \frac{\partial P\left(X-\Delta X, \Delta X ; t, t^{\prime}\right)}{\partial t} f\left(X-\Delta X, t^{\prime}\right) .
$$

Putting then $t^{\prime} \rightarrow t$ one obtains

$$
\begin{aligned}
\frac{\partial f(X, t)}{\partial t} & =\left.\int \mathrm{d} \Delta X \frac{\partial P\left(X-\Delta X, \Delta X ; t, t^{\prime}\right)}{\partial t}\right|_{t^{\prime} \rightarrow t} f\left(X^{\prime}, t\right) \\
& =\left.\int \mathrm{d} \Delta X \frac{\partial P(X-\Delta X, \Delta X ; t+\tau, t)}{\partial \tau}\right|_{\tau \rightarrow 0} f\left(X^{\prime}, t\right)
\end{aligned}
$$

that leads to the Fokker-Planck equation with time-dependent kinetic coefficients [8].

Obviously, equations (2) and (5) are derived under the assumption that $f(X, t)$ is not changed considerably during the time $\tau \sim \tau_{\mathrm{ph}} \gg \tau_{\text {cor }}$. Here, $\tau_{\mathrm{ph}}$ is the physically infinitesimal time over which the local time averaging of microscopic quantities is performed and with respect to which the relevant distribution function $f(X, t)$ and the displacement probability $P\left(X, \Delta X ; t, t^{\prime}\right)$ are introduced. The above assumption is correct if the characteristic time of the distribution function change $\tau_{\text {rel }}$ satisfies the condition $\tau_{\text {rel }} \gg \tau \sim \tau_{\text {ph }} \gg \tau_{\text {cor }}$. However, equations (2), (5) are no longer valid for the fast varying function $f(X, t)$.

In order to describe the evolution of $f(X, t)$ under the condition that $\tau_{\text {rel }}$ and $\tau_{\mathrm{ph}}$ are comparable, it is natural to introduce an equation that gives the relationship between $f(X, t)$ and its time derivative, namely

$$
f(X, t)=f\left(X, t_{0}\right)+\int_{t_{0}}^{t} \mathrm{~d} t^{\prime} \int \mathrm{d} \Delta X P\left(X-\Delta X, \Delta X ; t, t^{\prime}\right) \frac{\partial f\left(X-\Delta X, t^{\prime}\right)}{\partial t^{\prime}},
$$

where $t_{0}$ is the initial time of the system evolution. Within the context of equation (6), the distribution function $f(X, t)$ for arbitrary time $t>t_{0}$ is treated as the initial density $f\left(X, t_{0}\right)$ and the density of particles which appear at point $X$ at 
time $t$ due to all possible transitions in the past. Together with the equation for $f(X, t)$ in terms of the initial distribution $f\left(X, t_{0}\right)$,

$$
f(X, t)=\int \mathrm{d} X^{\prime} P\left(X-\Delta X, \Delta X, t, t_{0}\right) f\left(X-\Delta X, t_{0}\right),
$$

equation (6) generates the relation

$$
\frac{\partial f(X, t)}{\partial t}=-\frac{\partial}{\partial t} \int_{t_{0}}^{t} \mathrm{~d} t^{\prime} \int \mathrm{d} \Delta X \frac{\partial P\left(X-\Delta X, \Delta X ; t, t^{\prime}\right)}{\partial t^{\prime}} f\left(X-\Delta X, t^{\prime}\right),
$$

or

$$
\begin{aligned}
\frac{\partial f(X, t)}{\partial t} & =\int_{0}^{t} \mathrm{~d} t^{\prime} \int \mathrm{d} \Delta X\left\{\left.\frac{\partial P\left(X-\Delta X, \Delta X ; t, t^{\prime}\right)}{\partial t}\right|_{t^{\prime}=t} \delta\left(t-t^{\prime}\right)\right. \\
& \left.-\frac{\partial^{2} P\left(X-\Delta X, \Delta X ; t, t^{\prime}\right)}{\partial t \partial t^{\prime}}\right\} f(X, t),
\end{aligned}
$$

which is a natural generalization of equation (5). In fact, since $P\left(X-\Delta X, \Delta X ; t, t^{\prime}\right)$ is the probability of particle displacement, the derivative over $t^{\prime}$ in the second term of equation (8) gives the density change at the point $X$ at the moment $t$ per unit interval of $t^{\prime}$ and that over $t$ gives the density change per unit interval of $t$. Thus, integrating over $t^{\prime}$ leads to the density change in the unit interval of $t$ due to all possible transitions in the past. The first term in equation (8) is needed to take up the contribution of the singularity at $t=t^{\prime}$, since the time derivative of the probability $P\left(X, \Delta X, t ; t^{\prime}\right)$ has a discontinuity at the point $t=t^{\prime}$. The sign of the second term in equation (8) shows that the contribution of the transitions to the density changes decreases as $t-t^{\prime}$ increases.

In the case of a stationary system,

$$
P\left(X, \Delta X, t, t^{\prime}\right)=P\left(X, \Delta X, t-t^{\prime}\right)
$$

and equation (7) could be written as

$$
\begin{aligned}
& \frac{\partial f(X, t)}{\partial t}= \\
= & -\frac{\partial}{\partial t}\left\{f(X, t)-\frac{\partial}{\partial t} \int_{t_{0}}^{t} \mathrm{~d} t^{\prime} \int \mathrm{d} \Delta X P\left(X-\Delta X, \Delta X, t-t^{\prime}\right) f\left(X-\Delta X, t^{\prime}\right)\right\} .
\end{aligned}
$$

Notice that equation (8) reduces to equation (5) under the condition

$$
\left.\left.\frac{\partial P\left(X-\Delta X, \Delta X ; t, t^{\prime}\right)}{\partial t}\right|_{t^{\prime} \rightarrow t} \gg \tau_{\text {rel }} \frac{\partial^{2} P\left(X-\Delta X, \Delta X ; t, t^{\prime}\right)}{\partial t \partial t^{\prime}}\right|_{t^{\prime} \rightarrow t} .
$$


Equation (8) yields the non-Markovian Fokker-Planck equation

$$
\begin{aligned}
& \frac{\partial f(X, t)}{\partial t}= \\
= & -\frac{\partial}{\partial X_{i}} \int_{t_{0}}^{t} \mathrm{~d} t\left\{-a_{i}\left(X_{i}, t, t^{\prime}\right) f\left(X, t^{\prime}\right)+\frac{1}{2} \frac{\partial}{\partial X_{j}}\left(b_{i j}\left(X, t, t^{\prime}\right) f\left(X, t^{\prime}\right)\right)\right\},
\end{aligned}
$$

where

$$
\begin{aligned}
a_{i}\left(X, t, t^{\prime}\right) & =\frac{\partial\left\langle\Delta X_{i}\right\rangle_{X, t, t^{\prime}}}{\partial t} \delta\left(t-t^{\prime}\right)-\frac{\partial^{2}\left\langle\Delta X_{i}\right\rangle_{X, t, t^{\prime}}}{\partial t \partial t^{\prime}} \\
b_{i j}\left(X, t, t^{\prime}\right) & =\frac{\partial\left\langle\Delta X_{i} \Delta X_{j}\right\rangle_{X, t, t^{\prime}}}{\partial t} \delta\left(t-t^{\prime}\right)-\frac{\partial^{2}\left\langle\Delta X_{i} \Delta X_{j}\right\rangle_{X, t, t^{\prime}}}{\partial t \partial t^{\prime}} \\
\left\langle\Delta X_{i}\right\rangle_{X, t, t^{\prime}} & =\int \mathrm{d} \Delta X \Delta X_{i} P\left(X, \Delta X, t, t^{\prime}\right), \\
\left\langle\Delta X_{i} \Delta X_{j}\right\rangle_{X, t, t^{\prime}} & =\int \mathrm{d} \Delta X \Delta X_{i} \Delta X_{j} P\left(X, \Delta X, t, t^{\prime}\right) .
\end{aligned}
$$

If $f(X, t)$ changes slowly, then equation (9) reduces to equation (3), but with the time-dependent kinetic coefficients

$$
\begin{aligned}
A_{i}(X, t) & =\frac{\partial\left\langle\Delta X_{i}\right\rangle_{t, t_{0}}}{\partial t}, \\
B_{i, j}(X, t) & =\frac{\partial\left\langle\Delta X_{i} \Delta X_{j}\right\rangle_{t, t_{0}}}{\partial t} .
\end{aligned}
$$

The kinetic coefficients become constants only in the case of a linear time dependence of mean displacements.

The general relations thus obtained can be used to describe time-nonlocal diffusion. In particular, applying equation (9) to the real space and assuming that external fields are absent we come $\left(\right.$ at $\left.t_{0}=0\right)$ to the non-Markovian diffusion equation of the type derived in [2]:

$$
\frac{\partial n(\mathbf{r}, t)}{\partial t}=\int_{0}^{t} \mathrm{~d} t^{\prime} D\left(t, t^{\prime}\right) \frac{\partial^{2} n\left(\mathbf{r}, t^{\prime}\right)}{\partial \mathbf{r}^{2}},
$$

where

$$
\begin{aligned}
D\left(t, t^{\prime}\right) & =\frac{1}{2} \frac{\partial\left\langle\Delta r^{2}\right\rangle_{t, t^{\prime}}}{\partial t} \delta\left(t-t^{\prime}\right)-\frac{1}{2} \frac{\partial^{2}\left\langle\Delta r^{2}\right\rangle_{t, t^{\prime}}}{\partial t \partial t^{\prime}} \\
& =D_{0} \delta\left(t-t^{\prime}\right)-\frac{1}{2} \frac{\partial^{2}\left\langle\Delta r^{2}\right\rangle_{t, t^{\prime}}}{\partial t \partial t^{\prime}}
\end{aligned}
$$

In the case of stationary diffusion we have

$$
D(\tau)=D_{0} \delta(\tau)+\frac{1}{2} \frac{\partial^{2}\left\langle\Delta r^{2}\right\rangle_{\tau}}{\partial \tau^{2}}, \quad \tau=t-t^{\prime}
$$


The relevant equation for the non-Markovian transition probability is given by

$$
\frac{\partial}{\partial t} W\left(\mathbf{r}, \mathbf{r}^{\prime}, t\right)=\int_{0}^{t} \mathrm{~d} t^{\prime} D\left(t-t^{\prime}\right) \frac{\partial^{2} W\left(\mathbf{r}, \mathbf{r}^{\prime}, t^{\prime}\right)}{\partial \mathbf{r}^{2}}
$$

with the initial condition

$$
W\left(\mathbf{r}, \mathbf{r}^{\prime}, 0\right)=\delta\left(\mathbf{r}-\mathbf{r}^{\prime}\right) .
$$

Equation (14) recovers the non-Markovian diffusion equation derived in [2] within the continuous time random walk theory.

In $k, \omega$-representation the solution of equation (14) is

$$
W_{\mathbf{k} \omega}=\frac{\mathrm{i}}{\omega+\mathrm{i} k^{2} D_{\omega}}
$$

where

$$
D_{\omega}=\int_{0}^{\infty} \mathrm{d} \tau \mathrm{e}^{\mathrm{i} \omega \tau} D(\tau)
$$

Now let us specify the time behaviour of the mean-square displacement. Having assumed that $\tau$-dependence is subdiffusive we can write

$$
\left\langle\Delta r^{2}\right\rangle_{\tau}=A \tau^{\alpha}, \quad 0<\alpha \leqslant 1
$$

In this case the quantities

$$
\begin{aligned}
\frac{\partial\left\langle\Delta r^{2}\right\rangle_{\tau}}{\partial \tau} & =\alpha A \tau^{\alpha-1} \\
\frac{\partial^{2}\left\langle\Delta r^{2}\right\rangle_{\tau}}{\partial \tau} & =\alpha(\alpha-1) A \tau^{\alpha-2}
\end{aligned}
$$

are divergent for $\tau \rightarrow 0$. A possible way to get rid of the singularities in equations (12), (13) is to introduce the cutoff time $\tau_{\min }$ that is the minimum time for which the diffusion is "switched on". Thus,

$$
D_{0}=\left.\frac{1}{2} \frac{\partial\left\langle\Delta r^{2}\right\rangle_{\tau}}{\partial \tau}\right|_{\tau \rightarrow 0}=\left.\frac{1}{2} \frac{\partial\left\langle\Delta r^{2}\right\rangle_{\tau}}{\partial \tau}\right|_{\tau=\tau_{\min }}=\frac{1}{2} \alpha A \tau_{\min }^{\alpha-1}
$$

and

$$
D(\tau)=D_{0}\left\{\delta(\tau)-\frac{1-\alpha}{\tau_{\min }}\left(\frac{\tau_{\min }}{\tau}\right)^{2-\alpha}\right\}
$$

The Fourier component of $D(\tau)$ is given by

$$
D_{\omega}=D_{0}\left\{1-(1-\alpha) \tau_{\min }^{1-\alpha}\left(\frac{1}{\tau^{2-\alpha}}\right)_{\omega}\right\}
$$


where

$$
\left(\frac{1}{\tau^{2-\alpha}}\right)_{\omega} \equiv \int_{0}^{\infty} \mathrm{d} \tau \mathrm{e}^{\mathrm{i} \omega \tau} \frac{1}{\tau^{2-\alpha}}=-\frac{1}{\alpha(1-\alpha)} \int_{0}^{\infty} \mathrm{d} \tau \mathrm{e}^{\mathrm{i} \omega \tau} \frac{\partial^{2} \tau^{\alpha}}{\partial \tau^{2}} .
$$

Integrating by parts and using the regularization introduced above, we have

$$
\begin{aligned}
\left(\frac{1}{\tau^{2-\alpha}}\right)_{\omega} & =-\frac{1}{\alpha(1-\alpha)}\left\{-\left.\frac{\partial \tau^{\alpha}}{\partial \tau}\right|_{\tau=\tau_{\min }}-\omega^{2} \int_{0}^{\infty} \mathrm{d} \tau \mathrm{e}^{\mathrm{i} \omega \tau} \tau^{\alpha}\right\}= \\
& =\frac{\tau_{\min }^{\alpha-\alpha}}{1-\alpha}\left\{1-\left(-\mathrm{i} \omega \tau_{\min }\right)^{1-\alpha} \Gamma(\alpha)\right\}
\end{aligned}
$$

and thus,

$$
D_{\omega}=D_{0}\left(-\mathrm{i} \omega \tau_{\min }\right)^{1-\alpha} \Gamma(\alpha) .
$$

In terms of the quantity $\tau_{\mathrm{D}}=\tau_{\min }[\Gamma(\alpha)]^{\frac{1}{1-\alpha}}$,

$$
\begin{aligned}
D_{\tau} & =D_{0} \delta(\tau)-H_{0}\left(\frac{\tau}{\tau_{\mathrm{D}}}\right)^{\alpha-2}, \\
D(\omega) & =D_{0}\left(-\mathrm{i} \omega \tau_{\mathrm{D}}\right)^{1-\alpha}
\end{aligned}
$$

in agreement with the result of [2]. Here

$$
H_{0}=D_{0} \frac{1-\alpha}{\tau_{\mathrm{D}} \Gamma(\alpha)} .
$$

Within the context of the Einstein formula we can predict the viscoelasticity coefficient to be given by

$$
\beta_{\omega}=\frac{T}{m D_{\omega}}=\frac{T}{m D_{0}}\left(-\mathrm{i} \omega \tau_{\mathrm{D}}\right)^{\alpha-1},
$$

which makes it possible to compare the theoretical predictions with the experimental data on the anomalous diffusion in complex fluids. Applications of the non-Markovian diffusion equation to the description of transport in turbulent plasmas is discussed in section 4 .

\section{Microscopic derivation of the kinetic equations with the non- Markovian collision term}

The equation for the microscopic phase density

$$
N(X, t)=\frac{1}{n} \sum_{i=1}^{N} \delta\left(X-X_{i}(t)\right), \quad X=(\mathbf{r}, \mathbf{v})
$$


is given by

$$
\left\{\frac{\partial}{\partial t}+\mathbf{v} \frac{\partial}{\partial \mathbf{r}}+\frac{e}{m} \mathbf{E}(\mathbf{r}, t) \frac{\partial}{\partial \mathbf{v}}\right\} N(X, t)=0
$$

Here $\mathbf{E}(\mathbf{r}, t)$ is a microscopic electric field. The kinetic equation for the distribution function $f(X, t)=\langle N(X, t)\rangle$ can be obtained from equation (20) by its statistical averaging

$$
\left\{\frac{\partial}{\partial t}+\mathbf{v} \frac{\partial}{\partial \mathbf{r}}+\frac{e}{m}\langle\mathbf{E}(\mathbf{r}, t)\rangle \frac{\partial}{\partial \mathbf{v}}\right\} f(X, t)=I,
$$

where

$$
I=-\frac{e}{m} \frac{\partial}{\partial \mathbf{v}}\langle\delta \mathbf{E}(\mathbf{r}, t) \delta N(X, t)\rangle .
$$

The equation for fluctuations reduces then to

$$
\left\{\hat{L}^{0}+\frac{e}{m} \delta \mathbf{E} \frac{\partial}{\partial \mathbf{v}}\right\} \delta N(X, t)=-\frac{e}{m} \delta \mathbf{E} \frac{\partial f}{\partial \mathbf{v}},
$$

where

$$
\hat{L}^{(0)}=\frac{\partial}{\partial t}+\mathbf{v} \frac{\partial}{\partial \mathbf{r}}+\frac{e}{m}\langle\mathbf{E}(\mathbf{r}, t)\rangle \frac{\partial}{\partial \mathbf{v}} .
$$

The formal solution of equation (23) is given by

$$
\delta N(X, T)=\delta N^{0}(X, t)-\frac{e}{m} \int_{0}^{t} \mathrm{~d} t^{\prime} \int \mathrm{d} X^{\prime} W_{m}\left(X, X^{\prime} ; t, t^{\prime}\right) \delta \mathbf{E}\left(\mathbf{r}^{\prime}, t^{\prime}\right) \frac{\partial f\left(X^{\prime}, t\right)}{\partial \mathbf{v}^{\prime}}
$$

Here $\delta N^{(0)}(X, t)$ is the fluctuation of the microscopic phase density due to direct particle transitions which satisfies the equation

$$
\left\{\hat{L}^{(0)}+\frac{e}{m} \delta \mathbf{E} \frac{\partial}{\partial \mathbf{v}}\right\} \delta N^{0}(X, t)=0
$$

$W_{m}\left(X, X^{\prime} ; t, t^{\prime}\right)$ is the "microscopic" probability of the particle transition from point $X^{\prime}$ to $X$ during the time interval $t^{\prime}-t$. Obviously, the equation for such probability is

$$
\left\{\hat{L}^{(0)}+\frac{e}{m} \delta \mathbf{E} \frac{\partial}{\partial \mathbf{v}}\right\} W_{m}\left(X, X^{\prime} ; t, t^{\prime}\right)=0
$$

with the initial condition $W_{m}\left(X, X^{\prime} ; t, t^{\prime}\right)=\delta\left(X-X^{\prime}\right)$. The solution of equation (26) can be written as

$$
W\left(X, X^{\prime} ; t, t^{\prime}\right)=\delta\left(X-X^{\prime}-\Delta X\left(X^{\prime}, t^{\prime} ; t\right)\right)
$$

where $\Delta X\left(X^{\prime}, t^{\prime} ; t\right)$ is a particle phase displacement in the course of its motion in the microscopic field. 
We now assume that the distribution function slowly changes within the spatial and velocity fluctuation scales. Then, combining equations (22), (24) and (26) it is possible to derive a kinetic equation with a non-Markovian collision term, i.e.

$$
\hat{L}^{(0)} f(X, t)=\frac{\partial}{\partial v_{i}} \int_{0}^{t} \mathrm{~d} t^{\prime}\left\{b_{i}\left(t, t^{\prime}, v\right) f\left(X, t^{\prime}\right)+\frac{\partial}{\partial v_{j}}\left[\tilde{D}_{i j}^{(v)}\left(t, t^{\prime}, v\right) f\left(X, t^{\prime}\right)\right]\right\},
$$

where

$$
\begin{aligned}
b_{i}\left(t, t^{\prime}, v\right) & =b_{i}^{(1)}\left(t, t^{\prime}, \mathbf{v}\right)+b_{i}^{(2)}\left(t, t^{\prime}, \mathbf{v}\right), \\
b_{i}^{(1)}\left(t, t^{\prime}, \mathbf{v}\right) & =\mathrm{i} \frac{4 \pi e^{2} n}{m} \int \frac{\mathrm{d} \omega}{2 \pi} \int \frac{\mathrm{d} \mathbf{k}}{(2 \pi)^{3}} \frac{k_{i}\left\langle W_{m \mathbf{k} \omega}^{*}(\mathbf{v})\right\rangle}{k^{2} \varepsilon(k, \omega)} \delta\left(t-t^{\prime}\right), \\
b_{i}^{(2)}\left(t, t^{\prime}, v\right) & =-\frac{\partial}{\partial \mathbf{v}}\left(\frac{e}{m}\right)^{2} \int \frac{\mathrm{d} \mathbf{k}}{(2 \pi)^{3}}\left\langle\delta E_{i}(t) \delta E_{j}\left(t^{\prime}\right)\right\rangle_{\mathbf{k}}\left\langle W_{m \mathbf{k}}\left(v, t, t^{\prime}\right)\right\rangle, \\
\tilde{D}_{i j}^{v}\left(t, t^{\prime} v\right) & =\left(\frac{e}{m}\right)^{2} \int \frac{\mathrm{d} \mathbf{k}}{(2 \pi)^{3}}\left\langle\delta E_{i}(t) \delta E_{j}\left(t^{\prime}\right)\right\rangle_{\mathbf{k}}\left\langle W_{m \mathbf{k}}\left(\mathbf{v}, t, t^{\prime}\right)\right\rangle, \\
\varepsilon(k, \omega) & =1-\mathrm{i} \frac{4 \pi e^{2} n}{m k^{2}} \int \mathrm{d} \mathbf{v}\left\langle W_{m \mathbf{k} \omega}(\mathbf{v})\right\rangle \mathbf{k} \frac{\partial f}{\partial \mathbf{v}}
\end{aligned}
$$

and

$$
\begin{aligned}
\left\langle W_{m \mathbf{k}}\left(\mathbf{v}, t, t^{\prime}\right)\right\rangle & =\int_{0} \mathrm{~d} \Delta X \mathrm{e}^{-\mathrm{i} \mathbf{k} \Delta \mathbf{r}} W\left(X+\Delta X, X ; t, t^{\prime}\right), \\
\left\langle W_{m \mathbf{k} \omega}(\mathbf{v})\right\rangle & =\int_{0}^{\infty} \mathrm{d} \tau \mathrm{e}^{\mathrm{i} \omega \tau}\left\langle W_{m \mathbf{k}}(\mathbf{v}, t ; t-\tau)\right\rangle .
\end{aligned}
$$

We can see that the collision integral is expressed in terms of the averaged transition probability $\left\langle W_{m}\left(X, X^{\prime} ; t, t^{\prime}\right)\right\rangle$ renormalized with regard to the fluctuation field influence on the particle orbits.

Using equation (26), it is possible to show that this transition probability satisfies an equation of type (28), namely:

$$
\begin{aligned}
& \hat{L}^{0}\left\langle W_{m}\left(X, X^{\prime} ; t, t^{\prime}\right)\right\rangle= \\
= & \frac{\partial}{\partial v_{i}} \int_{t^{\prime}}^{t} \mathrm{~d} t^{\prime \prime}\left\{b_{i}\left(t, t^{\prime \prime}\right) W\left(X, X^{\prime} ; t^{\prime \prime}, t^{\prime}\right)+\frac{\partial}{\partial v}\left[D_{i j}^{v}\left(t^{\prime \prime}, t^{\prime}, v\right) W\left(X, X^{\prime} ; t^{\prime \prime}, t^{\prime}\right)\right]\right\} .
\end{aligned}
$$

Using equations (24), (25) we also find that

$$
\left\langle\delta E^{2}\right\rangle_{\mathbf{k} \omega}=\frac{16 \pi^{2}\left\langle\delta \rho^{2}\right\rangle_{\mathbf{k} \omega}^{(0)}}{|\varepsilon(k, \omega)|^{2}}
$$

where

$$
\left\langle\delta \rho^{2}\right\rangle_{\mathbf{k} \omega}^{(0)}=e^{2} n \int \mathrm{d} \mathbf{v} \int \mathrm{d} \mathbf{v}^{\prime}\left\langle\delta N^{(0)}(v) \delta N^{(0)}\left(v^{\prime}\right)\right\rangle_{\mathbf{k} \omega} .
$$

Taking into account equation (25), the latter relation can be rewritten as

$$
\left\langle\delta \rho^{2}\right\rangle_{\mathbf{k} \omega}^{(0)}=e^{2} n \int \mathrm{d} \mathbf{v}\left\{f(X, t)\left\langle W_{m k \omega}(\mathbf{v})\right\rangle+\text { c.c. }\right\} .
$$




\section{Non-Markovian transport and saturation of turbulence}

The next problem is to solve an equation for the transition probability. In variuos particular cases its solutions can be found explicitly. Substituting these solutions into equations (28) it is possible to derive self-consistent equations for the kinetic coefficients and thus to find the collision term. In particular, if the influence of fluctuation fields on the particle trajectory can be neglected,

$$
\left\langle W_{m \mathbf{k}}\left(\mathbf{v}, t, t^{\prime}\right)\right\rangle=\mathrm{e}^{\mathrm{i} \mathbf{k v}\left(t-t^{\prime}\right)}
$$

and $f\left(X, t^{\prime}\right) \simeq f(X, t)$, then equation (27) is reduced to the Balescu-Lenard equation, provided the electric field correlations are determined by the thermal particle motion (equations (32), (33)). For electric field fluctuations of the turbulent nature, equation (28) reproduces the result of the quasilinear theory $[9,10]$.

In the Markovian approximation, $\left(\left\langle W\left(X, X^{\prime} ; t^{\prime \prime}, t^{\prime}\right)\right\rangle\right.$ in equation $(31)$ is replaced by $\left.<W\left(X, X^{\prime} ; t, t^{\prime}\right)>\right)$ and for the nonstationary one-dimensional case we have:

$$
\left\langle W_{m \mathbf{k}}\left(v, t, t^{\prime}\right)\right\rangle=\exp \left[\mathrm{i} \frac{k v}{\beta}\left(1-\mathrm{e}^{-\beta \tau}\right)-\frac{k^{2}}{\beta^{2}} \int_{0}^{\tau} \mathrm{d} \xi D^{v}(t-\xi)\left(1-\mathrm{e}^{-\beta(\tau-\xi)}\right)^{2}\right],
$$

where

$$
D^{v}(t)=\int_{0}^{t} \mathrm{~d} t^{\prime} \tilde{D}^{v}\left(t, t^{\prime}, v\right) ; \quad \beta=\int_{0}^{t} \mathrm{~d} t^{\prime} b\left(t, t^{\prime}, v\right) ; \quad \tau=t-t^{\prime} .
$$

In the stationary case for $\beta \tau \ll 1$, equation (35) reduces to

$$
\left\langle W_{m \mathbf{k}}\left(v, t, t^{\prime}\right)\right\rangle=\exp \left[\mathrm{i} k v \tau-\frac{k^{2} D^{v} \tau^{3}}{3}\right]
$$

which leads to the results of the Dupree-Weinstock theory [11,12] after substituting equation (36) into equation (28) at $f\left(X, t^{\prime}\right) \simeq f(X, t)$.

In the opposite limiting case, $\beta \tau \gg 1$, we obtain

$$
\left\langle W_{m \mathbf{k}}\left(v, t, t^{\prime}\right)\right\rangle=\exp \left(-k^{2} D \tau\right),
$$

which corresponds to integration along the diffusive orbits. Here $D=D^{v} / \beta^{2}$ in the diffusion coefficient in the real space.

The solution of equation (31) in the non-Markovian one-dimensional case generates an equation for the Fourier-component of the velocity diffusion coefficient, i.e.

$$
\begin{aligned}
\tilde{D}_{\omega}^{v} & =\left(\frac{e}{m}\right)^{2} \int_{0}^{\infty} \mathrm{d} \tau \mathrm{e}^{\mathrm{i} \omega \tau} \int \frac{\mathrm{d} k}{2 \pi}\langle\delta E(t) \delta E(t-\tau)\rangle \\
& \times \int \frac{\mathrm{d} \omega_{1}}{2 \pi} \mathrm{e}^{\mathrm{i} \omega_{i} \tau} \int_{0}^{\infty} \mathrm{d} \tau_{1} \mathrm{e}^{-\mathrm{i} \omega \tau_{1}} \exp \left[\frac{\mathrm{i} k v}{b_{\omega_{1}}}-\frac{k^{2} \tilde{D}_{\omega_{1}}^{v}}{b_{\omega_{1}}^{2}}\right] .
\end{aligned}
$$


Here $\tilde{D}_{\omega}^{v}$ and $b_{\omega}$ are the Fourier-components of the quantities $\tilde{D}^{v}\left(t, t^{\prime}, v\right)$ and $b\left(t, t^{\prime}, v\right)$, respectively. This equation makes it possible to estimate the saturation level and diffusion coefficients for the turbulence generated by plasma instabilities. In particular, for the model turbulent spectrum,

$$
\langle\delta E(\mathbf{r}+\mathbf{R}, t+\tau) \delta E(\mathbf{r}, t)\rangle=\delta E_{0}^{2} \cos \left(k_{0} R-\omega_{0} \tau\right) .
$$

Equation (38) yields a relation for the turbulent diffusion coefficient in the coordinate space $\left(b_{\omega} \tau \gg 1\right)$. Thus we have:

$$
D_{\omega}=\left(\frac{e}{m}\right)^{2} \frac{\delta E_{0}^{2}}{2 b_{\omega}^{2}} \sum_{m= \pm 1} \frac{\mathrm{i}}{\omega-m \omega_{0}+\mathrm{i} k^{2} D_{n \omega_{0}-\omega}} .
$$

If the turbulence saturation is obtained due to the diffusive compensation of the linear growth rate $\gamma_{\mathbf{k}}$ (that is the case for the gradient-driven instability), equation (39) yields an estimate of the static value of the diffusion coefficient,

$$
D_{0}=\frac{\gamma_{k}^{3} / k^{2}}{\omega_{0}^{2}+\gamma_{k}^{2}}
$$

which is in agreement with the observations and mode coupling simulations [6]. This estimate could not be obtained within the Markovian approximation.

\section{Conclusions}

Using the assumption that the physically infinitesimal time (which defines the averaged microscopic quantities) and the correlation time of random forces are comparable and introducing the integral relation between the distribution function and its time derivative, we obtain a Fokker-Planck-type equation with regard to the memory effects.

The kinetic coefficients in this equation are expressed in terms of the displacement moments of the relevant probability function. Application of the proposed equation to the case of three-dimensional coordinate space generates a timenonlocal (non-Markovian) diffusion equation derived earlier within the continuous random walk theory [2]. The equations with time-nonlocal diffusion terms and the general relations for the kinetic coefficients make it possible to study the evolution of non-Markovian systems, provided that mean and mean-square displacements are known $[4,5]$.

The microscopic derivation of the kinetic equation with a non-Markovian collision term is done for the case of turbulent plasmas. For this purpose the transition probability approach is modified to the case under consideration. Equations for the renormalized transition probability are formulated with regard to the turbulent field influence on the particle trajectories. In the appropriate approximation the results of the quasilinear theory $[9,10]$, as well as of the Dupree-Wienstock theory $[11,12]$, are reproduced. The transition probability for a stationary non-Markovian 
system is found as well, and the estimates for the time-nonlocal diffusion coefficients in both real and velocity spaces are obtained.

It is shown that the memory effects can be important for the description of transport under saturated turbulence. In particular, time nonlocality of the diffusion coefficients can be used to explain the dependence of the transport properties of saturated turbulence on the eigenfrequency of the unstable mode in the case of instability driven by the gradients in the coordinate space.

\section{Acknowledgements}

The authors acknowledge financial support within the INTAS Cooperation Projects 95-0133, 96-0617, and the Projects of the State Fund of Fundamental Studies of Ukraine (Grant No2.4/319 and 2.5.2/46).

\section{References}

1. Xia H., Ishihara O., Hirose A. // Phys. Fluids B, 1993, vol. 5, p. 2892.

2. Balescu R. // Phys. Rev. E, 1995, vol. 51, p. 4807.

3. Zagorodny A., Weiland J., Jarmen A. // Comments on Plasma Phys. Controlled Fusion, 1997, vol. 17, p. 353.

4. Mason T.G., Weitz D.A. // Phys. Rev. Lett., 1995, vol. 74, p. 1250.

5. Barkai E., Fleurov V.N., Klafter J. // Response of Anomalous Diffusion Processes to an External Field. In: 20-th IUPAP Conference on Statistical Physics (Paris, July 19-23, 1998). Book of Abstracts. Paris, 1998, p. 3 (part SC02).

6. Nordman H., Weiland J. // Nuclear Fusion, 1989, vol. 29, p. 251.

7. Chandrasekhar S. // Rev. Mod. Phys., 1943, vol. 15, p. 1.

8. Klimontovich Yu.L. // Turbulent Motion and Structure of Chaos. Moscow, Nauka, 1990 (in Russian).

9. Vedenov A.A., Velikhov E.P., Sagdeev R.Z. // Nucl. Fusion Suppl. Pt. 2, 1962, p. 465.

10. Drummond W.E. and Pines D. // Nucl. Fusion Suppl. Pt. 3, 1962, p. 1049.

11. Dupree T.H. // Phys. Fluids, 1976, vol. 10, p. 1049.

12. Weinstock J. // Phys. Fluids, 1969, vol. 12, p. 1045. 


\title{
Немарковські рівняння Фокера-Планка і турбулентне перенесення в плазмі
}

\author{
А.Г.Загородній ${ }^{1}$, Я.Вейланд ${ }^{2}$ \\ 1 Інститут теоретичної фізики ім. М.М.Боголюбова НАН України, \\ 252143 Київ 143 \\ 2 Інститут електромагнетизму Чалмерського технічного університету, \\ S-41296, Ґетеборґ, Швеція
}

Отримано 6 травня 1998 р.

3 метою опису впливу “пам'яті” на стохастичну динаміку частинок у реальному просторі та просторі швидкостей запропоновано немарківське узагальнення рівняння Фокера-Планка. Встановлено загальні співвідношення між часово-нелокальними коефіцієнтами дифузії, іхніми звичайними складовими та середньоквадратичними зміщеннями. Застосування запропонованих рівнянь до опису дифузії в реальному просторі дає змогу ввести рівняння дифузії з часово-нелокальними коефіцієнтами.

Мікроскопічне виведення немарковських рівнянь Фокера-Планка дано для випадку турбулентної плазми з урахуванням часової еволюції кінетичних коефіцієнтів. Обговорюється вплив ефектів часової нелокальності кінетичних коефіцієнтів на насичення плазмової турбулентності.

Ключові слова: рівняння Фокера-Планка, насичення турбулентності, часово-нелокальна дифузія

PACS: 05.40. $+j ;$ 52.35.Ra; 25.65. Ff 
